\title{
Nucleation Kinetics on Inhomogeneous Substrates: $\mathrm{Al} / \mathrm{Au}(111)$
}

\author{
Bjørn Fischer, Harald Brune, Johannes V. Barth, Alexander Fricke, and Klaus Kern \\ Institut de Physique Expérimentale, EPF Lausanne, CH-1015 Lausanne, Switzerland
}

(Received 29 June 1998)

\begin{abstract}
We report a quantitative atomic scale study of nucleation kinetics on an inhomogeneous substrate. Our model system, $\mathrm{Al} / \mathrm{Au}(111)-(\sqrt{3} \times 22)$, reveals a distinct nucleation transition due to the repulsive nature of surface dislocations. Whereas for $T<200 \mathrm{~K} \mathrm{Al}$ adatoms are confined to quasipseudomorphic stacking areas experiencing a very small diffusion barrier $(30 \pm 5 \mathrm{meV})$, at $T>200 \mathrm{~K}$ surface dislocations, representing repulsive barriers of $\Delta E \approx 560 \mathrm{meV}$, can be surmounted. The results illustrate the significance of surface dislocations as repulsive line defects in nucleation and growth. [S0031-9007(99)08540-3]
\end{abstract}

PACS numbers: 68.55.-a, 61.16.Ch, 68.35.Fx, 81.10.Aj

Molecular beam epitaxy of semiconductors and metals is frequently governed by the growth kinetics being determined by the competition between the adatom diffusion rates and the deposition flux [1]. Recent scanning tunneling microscopy (STM) studies established the link between the activation energies of the related atomic displacement processes and the resulting island densities, shapes, and film morphologies [2]. Because of these experimental and various theoretical studies our understanding of the initial stages of thin film growth on both isotropic and anisotropic substrates improved considerably. These substrates are homogeneous since adatom diffusion occurs exclusively by jumps between simple unit cells, i.e., the binding and diffusion energies remain invariant when the atom migrates.

However, many surfaces in nature show large scale reconstructions, either in their clean state or they restructure during growth. In heteroepitaxy this restructuring is driven by strain often inducing complex patterns of ordered partial surface dislocations or moiré structures [3]. Such surfaces belong to a class that we call inhomogeneous, since their structure and with it also their adatom binding and diffusion energies change on a much larger length scale than that of individual lattice jumps. Therefore the diffusing adatom experiences a complicated potential energy surface, where the migration barrier and the degree of diffusion anisotropy depend on its position within the large scale unit cell. While nucleation on homogeneous substrates has been extensively addressed in experiment and theory, a quantitative investigation of diffusion and nucleation on inhomogeneous substrates is presently lacking. Several observations reported in the literature [4] suggest that dislocations can represent considerable repulsive barriers towards diffusing adatoms by which they may control nucleation and growth.

In this Letter we present the first quantitative study of the impact of surface dislocations on adatom diffusion and nucleation kinetics. We studied Al heteroepitaxy on $(\sqrt{3} \times 22)$-reconstructed $\mathrm{Au}(111)$ [5] as a function of temperature by means of variable temperature STM. In contrast to earlier room temperature studies with $\mathrm{Ni}$, $\mathrm{Fe}$, or $\mathrm{Co}[6]$, we observe that the reconstruction lines are repulsive towards diffusing $\mathrm{Al}$ adatoms, and that there is no preferred nucleation at the elbows of the chevron reconstruction. Recent results for $\mathrm{Ni} / \mathrm{Au}(111)$ indicate that the preferential nucleation at elbows is due to thermally activated surface atom exchange [7]. With the present experiments the pure adatom diffusion at low substrate temperature is studied.

The experiments were performed in a UHV chamber equipped with a variable temperature STM [8]. The $\mathrm{Au}(111)$ single crystal surface was prepared by cycles of $\mathrm{Ar}^{+}$sputtering $\left(1 \mu \mathrm{A} / \mathrm{cm}^{2}, 700 \mathrm{eV}\right)$ at 300 and $1000 \mathrm{~K}$ annealing [5]. Al was evaporated in situ from a Knudsen cell at background pressures below $5 \times 10^{-10}$ mbar. Auger electron spectroscopy revealed the absence of contaminations for $\mathrm{Al}$ films as thick as $10 \mathrm{ML}$ [1 monolayer (ML) corresponds to $1 \mathrm{Al}$ atom per $\mathrm{Au}$ substrate atom]. STM data were acquired isothermally to or at lower temperatures than deposition.

A series of STM images characterizing the submonolayer nucleation of $\mathrm{Al}$ on $\mathrm{Au}(111)$ as a function of substrate temperature is reproduced in Fig. 1. The island density decreases with increasing deposition temperature due to the increasing mean free path of diffusing adatoms prior to creation of a nucleus. This decrease is discontinuous with three distinct nucleation regimes. Up to $200 \mathrm{~K}$ large temperature changes induce only small variations of the island density. The islands are homogeneously distributed on the substrate terraces, in contrast to observations of site specific nucleation [6,7]. The island density then drops rapidly in the range from 200 to $245 \mathrm{~K}$. For temperatures exceeding $245 \mathrm{~K}$, a third nucleation regime is identified by an even stronger island density falloff with temperature. Each of the three nucleation regimes exhibits Arrhenius behavior, which is reflected by three different slopes in the corresponding representation of the island densities in Fig. 1(f). The island densities were acquired at $0.10-0.15 \mathrm{ML}$ and thus represent saturation island densities $n_{x}$ [9]. On isotropic substrates $n_{x}$ is related at low temperatures to the ratio of the surface diffusion constant $D$ to the deposition flux $F$ by the scaling law $n_{x}=\eta(D / F)^{-1 / 3}$ (result from mean-field nucleation 
a) $150 \mathrm{~K}$

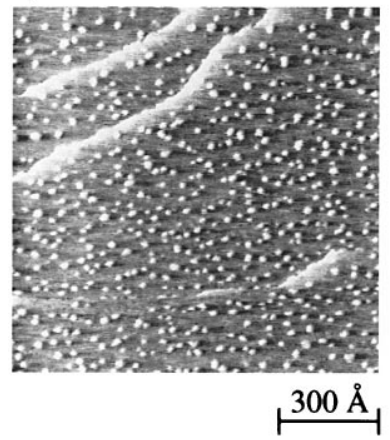

c) $235 \mathrm{~K}$

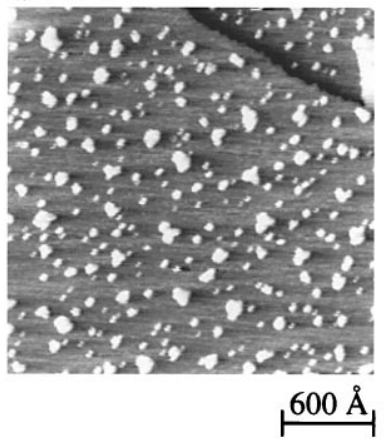

e) $260 \mathrm{~K}$

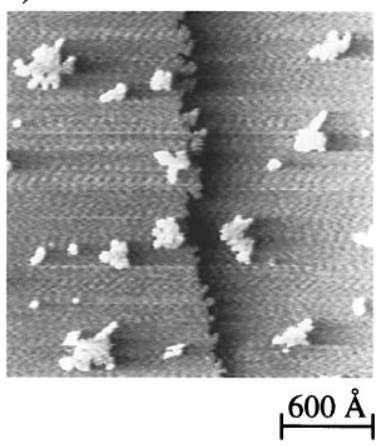

b) $200 \mathrm{~K}$

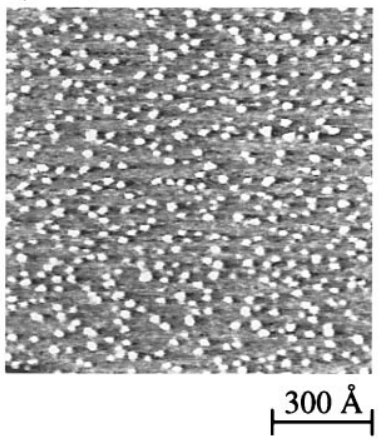

d) $250 \mathrm{~K}$
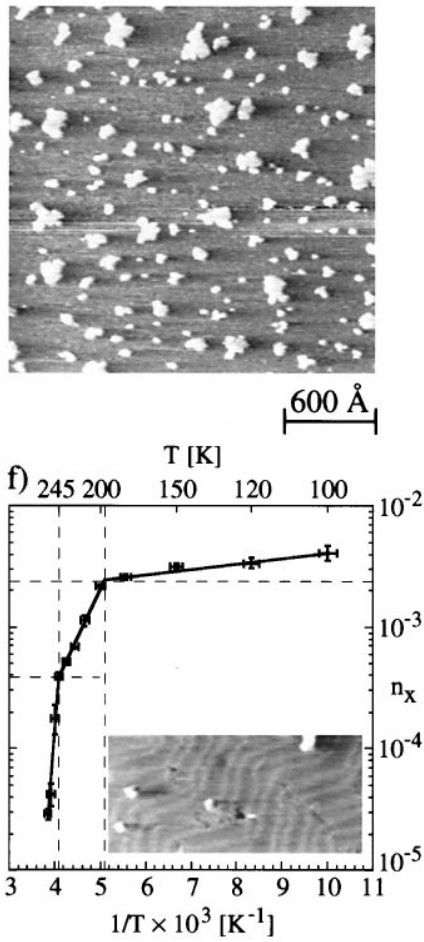

FIG. 1. STM images [(a)-(e)] showing the evolution of island density, size, and shape as a function of temperature for $\mathrm{Al} \mathrm{de}$ position onto $\mathrm{Au}(111)\left[\Theta=0.1 \mathrm{ML}, F=3.1 \times 10^{-4} \mathrm{ML} / \mathrm{s}\right.$; because of the large scale the substrate reconstruction is not resolved; note the change in scale between (b) and (c)]. (f) Arrhenius plot of saturation island densities $n_{x}$ (in units of islands per lattice site) derived from a statistical analysis of a series of STM images for each temperature. The inset reveals the coexistence of the $\mathrm{Au}(111)$ reconstruction with $\mathrm{Al}$ adatom islands at $T=150 \mathrm{~K}(\Theta=0.04 \mathrm{ML})$.

theory for a critical cluster size $i=1$, where $\eta \approx 0.25$ $[2,9])$. Whereas the lowest-temperature branch in the Arrhenius plot can be reconciled with such a picture (see below), the rapid island density falloffs at $T=200 \mathrm{~K}$ and $T=245 \mathrm{~K}$ cannot be understood in terms of nucleation on a homogeneous substrate. This signals thermal activation of other atomic processes.

The determination of the respective substrate structures and island size distributions provides additional insight into the nucleation transitions: The $\mathrm{Au}(111)$ chevron structure prevails for $\mathrm{Al}$ deposition up to $240 \mathrm{~K}$ [cf. Fig. 1(f)]. Accordingly, helium atom scattering (HAS) reveals an unperturbed $\mathrm{Au}(111)-(\sqrt{3} \times 22)$ diffraction pattern for $\mathrm{Al}$ deposition up to $230 \mathrm{~K}$ [10]. For adsorption below this temperature the island size distributions closely resemble those for a critical nucleus size of $i=1$ [cf. Fig. 2(a)] known from scaling theory and simulations on isotropic and anisotropic lattices [11]. Since density-functional theory also demonstrates high stability of $\mathrm{Al}$ dimers $\left[E_{b} \approx\right.$ $0.53-0.56 \mathrm{eV}$ on $\mathrm{Al}(111)$ ] [12], we conclude that $i=1$ for $T<230 \mathrm{~K}$. Hence the drastic change in slope by a factor of 16 at $T=200 \mathrm{~K}$ is not due to a change in critical nucleus size. Rather it must be related to a change in the diffusivity of adatoms. In contrast, for $T \geq 245 \mathrm{~K}$ a substrate symmetry change occurs. STM data reveal a poorly ordered dislocation pattern with sixfold symmetry [13], again corroborated by HAS [10]. The island size distributions for $T \geq 245 \mathrm{~K}$ are bimodal [cf. Fig. 2(b)]. They are dominated by the curve expected for $i=0$ which signals surface atom exchange [14], eventually leading to $\mathrm{Al}-\mathrm{Au}$ surface alloy formation [13].

Since the reconstruction remains unperturbed below $\approx 240 \mathrm{~K}$ the diffusivity change at $200 \mathrm{~K}$ must have an origin, which escapes from standard descriptions of nucleation and growth. Our data indicate that the aluminum islands are mostly located in the quasipseudomorphic stacking areas, off from the $\mathrm{Au}(111)$ reconstruction corrugation lines. Apart from this, however, there is no preferred nucleation at any particular site [see the homogeneous spatial distribution of islands in Figs. 1(a) and 1(b)]. These observations signal that the partial surface dislocations of the reconstruction repel diffusing $\mathrm{Al}$ adatoms. The 200-K change in slope in the Arrhenius representation of $n_{x}$ appears at a density, where the mean island distance is 22 atoms [upper dashed horizontal line in Fig. 1(f)]. This corresponds to the periodicity of the reconstruction and thus suggests an increase in diffusivity related to
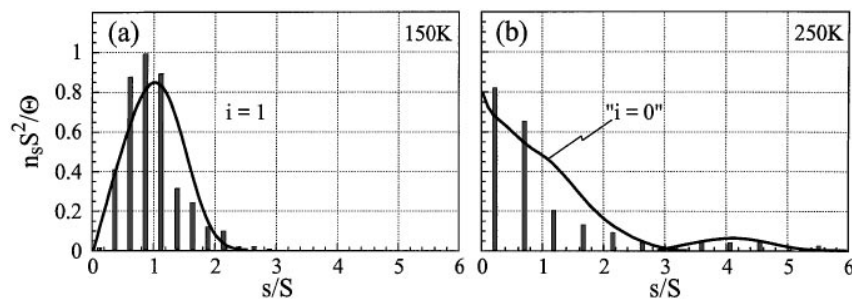

FIG. 2. Normalized island size distributions from STM data. (a) Pure adatom nucleation with $i=1$ scaling on the naturally reconstructed $\mathrm{Au}(111)$ surface for temperatures up to $230 \mathrm{~K}$. Island size distributions are compared to scaling theory for isotropic lattices (curve). On anisotropic lattices, the maximum shifts slightly to the left and its height increases [11], trends which agree well with the present distributions. (b) Bimodal island size distribution dominated by an $i=0$ scaling behavior induced by substrate structural transformation and surface atom exchange for temperatures exceeding $245 \mathrm{~K}$. 
diffusion over dislocations representing repulsive barriers. Below $200 \mathrm{~K}$, where the island density is high, $\mathrm{Al}$ adatoms are hence confined to the quasipseudomorphic stacking areas. Whenever they move towards a dislocation they are reflected, however, they are free to move parallel to the dislocations. Above $200 \mathrm{~K}$ the island density reaches a threshold, where adatoms must have diffused over dislocations which leads to a change in the effective diffusion barrier.

A straightforward analysis of the Arrhenius plot of $n_{x}$ in terms of scaling laws is obstructed by the inhomogeneous nature of the substrate. There are mean-field models for heterogeneous nucleation in the presence of attractive or repulsive point defects [15], however, for the repulsive line defects in the present system there is no theoretical treatment so far. Hence we adopted a kinetic Monte Carlo (KMC) model [16] to simulate diffusion and nucleation on the complex potential energy surface experienced by an $\mathrm{Al}$ adatom on the reconstructed $\mathrm{Au}(111)$ surface. The model incorporates two partial dislocations per $(\sqrt{3} \times 22)$ unit cell [Figs. 3(b) and 3(c)]. Effective medium theory calculations for $\mathrm{Pt} / \mathrm{Pt}(111)$ [17] indicate that the adatom potential is affected within a range of several atomic distances around the dislocations. Accordingly, we selected the barriers for diffusing up, $E_{\mathrm{d} l \uparrow}$, or down, $E_{\mathrm{d} l \downarrow}$, a dislocation in

a)

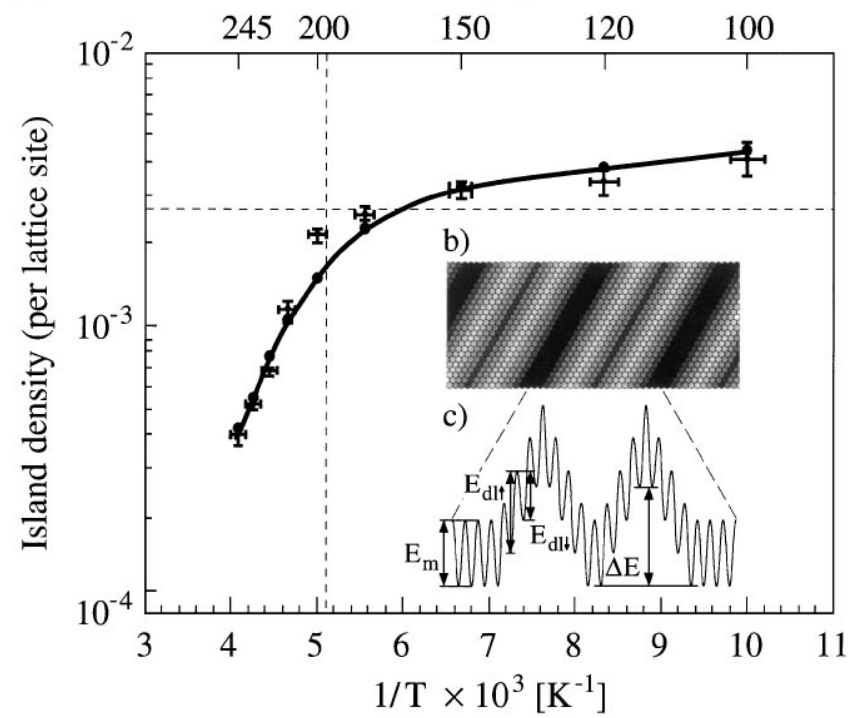

FIG. 3. (a) Comparison of experimental island densities to KMC simulations. A good fit is obtained for $E_{m}=30 \mathrm{meV}$ $\left(\nu_{0}=2 \times 10^{3} \mathrm{~s}^{-1}\right), \quad E_{\mathrm{d} 1 \uparrow}=350 \mathrm{meV} \quad\left(\nu_{0}=2 \times 10^{13} \mathrm{~s}^{-1}\right)$, and $E_{\mathrm{d} \downarrow}=210 \mathrm{meV}\left(\nu_{0}=2 \times 10^{11} \mathrm{~s}^{-1}\right)$. (b) and $(c)$ : The KMC model incorporates two partial dislocations as line defects over \pm 4 sites. For simplicity, in the model the dislocations run along the close-packed atomic rows. For an atom at a dislocation we select $E_{m}$ for the two jump directions parallel to the dislocation, $E_{\mathrm{d} 1 \uparrow}$ for the two jumps towards the dislocation, and $E_{\mathrm{dll}}$ for the two jumps away from the dislocation; in the quasipseudomorphic stacking $E_{m}$ holds in all jump directions (with corresponding $\nu_{0}$, respectively). a range of \pm 4 atomic distances left and right from the dislocation center. For adatoms in the quasipseudomorphic stacking areas, and for jump directions parallel to the dislocations, we selected the barrier for terrace diffusion $E_{m}$. The diffusion barrier therefore depends both on the adatom position and the jump direction, a general feature of inhomogeneous substrates. Different attempt frequencies $\nu_{0}$ can be associated with each atomic displacement process.

Using this KMC model a systematic search was performed to find an optimal parametrization providing a satisfying fit to the Arrhenius plot in the range of $100 \leq$ $T \leq 245 \mathrm{~K}$ [see Fig. 3(a)]. Although the transition between the two slopes at $200 \mathrm{~K}$ is less abrupt in the simulations, the asymptotic slopes agree well with the experimental line fits. The main results deduced from the simulations are as follows. Three diffusion rates are involved, the rate to move up, $D_{\mathrm{d} 1 \uparrow}$, or to descend a dislocation, $D_{\mathrm{d} 1 l}$, and the terrace diffusion rate, $D$. As islands are not observed on top of dislocations, $D_{\mathrm{d} 1 \downarrow}$ must be the largest rate at all temperatures enabling adatoms deposited on top of dislocations to descend. The change in slope at $200 \mathrm{~K}$ implies a continuous transition in the rate limiting diffusion process, from $D>D_{\mathrm{d} 1 \uparrow}$ at $T \leq 200 \mathrm{~K}$ to $D<D_{\mathrm{d} 1 \uparrow}$ at $T>200 \mathrm{~K}$. Up to $200 \mathrm{~K}$ the adatom motion is hence dominated by $D$, and the slope of $\log n_{x}$ vs $1 / T$ directly yields the barrier for Al diffusion in the quasipseudomorphic stacking areas of $\mathrm{Au}(111)$, which is determined from the KMC simulations to $E_{m}=30 \mathrm{meV}$. This rather small value compares well with the $a b$ initio result $E_{m}=40 \mathrm{meV}$ for $\mathrm{Al}(111)$ self-diffusion [12]. The absolute values of the island densities observed in experiment imply a surprisingly small prefactor for $\mathrm{Al}$ diffusion on $\mathrm{Au}(111)$ of $\nu_{0}=2 \times 10^{3} \mathrm{~s}^{-1}$ [18]. Analyzing the slope (and intersection) within mean-field nucleation theory yields an identical barrier of $E_{m}=30 \pm 5 \mathrm{meV}$ (and a prefactor of $\nu_{0}=7 \times 10^{3 \pm 1} \mathrm{~s}^{-1}$ ). A mean-field treatment of anisotropic diffusion yields a slightly larger barrier of $E_{m}=40 \pm 7 \mathrm{meV}$. The agreement of both mean-field values with that from the KMC model indicates that for $T<200 \mathrm{~K}$ the system is well described by a homogeneous model with quasi-isotropic diffusion being rate limiting. Apparently, the occasional reflections at dislocations do not significantly distort the density scaling. By contrast, the slope for $T>200 \mathrm{~K}$ reflects the perturbation of diffusion due to the dislocations. It is parametrized in our model by $E_{\mathrm{d} l \uparrow}=350 \mathrm{meV}\left(\nu_{0}=2 \times 10^{13} \mathrm{~s}^{-1}\right)$, and $E_{\mathrm{d} 1 \downarrow}=210 \mathrm{meV} \quad\left(\nu_{0}=2 \times 10^{11} \mathrm{~s}^{-1}\right)$, respectively. The effective barrier for dislocation crossing is the difference in total adatom binding energy between quasipseudomorphic sites and adsorption sites on top of the dislocation. This energy difference amounts to $\Delta E=4\left(E_{\mathrm{d} 1 \uparrow}-E_{\mathrm{d} 1 \downarrow}\right)=560 \mathrm{meV}$ in accordance with the 16 times larger slope observed above $200 \mathrm{~K}$ [19].

The multitude of atomic processes entering through the substrate transformation and adatom exchange at $T>$ $240 \mathrm{~K}$ obscure a quantitative analysis of this regime. 
Nevertheless, the bimodal size distribution [Fig. 2(b)] can be interpreted qualitatively. The peak corresponding to large island sizes is reminiscent of an additional nucleation mechanism taking place in the very initial stage of deposition. Since the $\mathrm{Al} / \mathrm{Au}(111)$ system is unstable towards alloy formation, it is attributed to preferential atom exchange at special sites [7]. Consequently the large islands nucleate in the beginning of the "transient regime," where the linearly increasing monomer density $n_{1}$ is very low [20]. The mean free path of $\mathrm{Al}$ adatoms is thus high and they easily attain the special sites. These early nuclei grow in the course of further deposition until with increasing monomer density nucleation of adatom islands sets in. This rapidly diminishes the mean free path. Hence nucleation and growth of adatom islands dominate in the "steady state regime" of the monomer density [20].

In conclusion, by analysis of temperature dependent island densities with realistic KMC simulations, the complex potential energy surface experienced by an $\mathrm{Al}$ adatom on the $\mathrm{Au}(111)-(\sqrt{3} \times 22)$ surface was derived. The repulsive nature of the partial dislocations of the $\mathrm{Au}(111)$ reconstruction leads to adatom confinement at low temperatures and drives a nucleation transition for temperatures exceeding $200 \mathrm{~K}$. It is believed that such effects are of general importance for heteroepitaxial growth or for homoepitaxial systems that are unstable towards reconstruction.

We gratefully acknowledge J. Jacobsen for providing us with the KMC code that we adopted to the inhomogeneous potential energy surface of $\mathrm{Au}(111)$. A. F. acknowledges financial support from the Alexander von Humboldt-Stiftung.

[1] C. Günther, S. Günther, E. Kopatzki, R. Q. Hwang, J. Schröder, J. Vrijmoeth, and R. J. Behm, Ber. Bunsenges. Phys. Chem. 97, 522 (1993); M. G. Lagally, Phys. Today 46, No. 11, 24 (1993).

[2] H. Brune, Surf. Sci. Rep. 31, 121 (1998), and references therein.

[3] H. Brune and K. Kern, in The Chemical Physics of Solid Surfaces and Heterogeneous Catalysis, edited by D. A. King and D. P. Woodruff (Elsevier Science, Amsterdam, 1997), Vol. 8, p. 149.

[4] J. A. Meyer, P. Schmid, and R. J. Behm, Phys. Rev. Lett. 74, 3864 (1995); H. Brune, K. Bromann, H. Röder, K. Kern, J. Jacobsen, P. Stolze, K. Jacobsen, and
J. Nørskov, Phys. Rev. B 52, R14380 (1995); T. Michely, M. Hohage, S. Esch, and G. Comsa, Surf. Sci. 349, L89 (1996).

[5] J. V. Barth, H. Brune, G. Ertl, and R. J. Behm, Phys. Rev. B 42, 9307 (1990); A. R. Sandy, S. G. J. Mochrie, D. M. Zehner, K. G. Huang, and D. Gibbs, Phys. Rev. B 43, 4667 (1991).

[6] D. D. Chambliss, R. J. Wilson, and S. Chiang, Phys. Rev. Lett. 66, 1721 (1991); B. Voigtländer, G. Meyer, and N. M. Amer, Surf. Sci. 255, L529 (1991); Phys. Rev. B 44, 10354 (1991).

[7] J. A. Meyer, J. D. Baikie, E. Kopatzki, and R. J. Behm, Surf. Sci. 365, L647 (1996).

[8] H. Brune, H. Röder, K. Bromann, and K. Kern, Thin Solid Films 264, 230 (1995).

[9] J. A. Venables, Philos. Mag. 17, 697 (1973).

[10] M. A. Krzyzowski, Ph.D. thesis, University of Bonn, 1995.

[11] J. G. Amar and F. Family, Phys. Rev. Lett. 74, 2066 (1995); T. R. Linderoth, J. J. Mortensen, K. W. Jacobsen, E. Lægsgaard, I. Stensgaard, and F. Besenbacher, Phys. Rev. Lett. 77, 87 (1996); J. J. Mortensen, T. R. Linderoth, K. W. Jacobsen, E. Lægsgaard, I. Stensgaard, and F. Besenbacher, Surf. Sci. 400, 290 (1997).

[12] R. Stumpf and M. Scheffler, Phys. Rev. Lett. 72, 254 (1994); Phys. Rev. B 53, 4958 (1996); A. Bogicevic, P. Hyldgaard, G. Wahnström, and B. Lundqvist, Phys. Rev. Lett. 81, 172 (1998).

[13] B. Fischer, J. V. Barth, A. Fricke, L. Nedelmann, and K. Kern, Surf. Sci. 389, 366 (1997).

[14] D. D. Chambliss and K. E. Johnson, Phys. Rev. B 50, 512 (1994).

[15] K. R. Heim, S. T. Coyle, G. G. Hembree, J. A. Venables, and M.R. Scheinfein, J. Appl. Phys. 80, 1161 (1996); S. Liu, L. Bönig, J. Detch, and H. Metiu, Phys. Rev. Lett. 74, 4495 (1995).

[16] J. Jacobsen, K. W. Jacobsen, P. Stoltze, and J. K. Nørskov, Phys. Rev. Lett. 74, 2295 (1995).

[17] J. Jacobsen, K. W. Jacobsen, and P. Stoltze, Surf. Sci. 317, 8 (1994).

[18] We systematically observe by orders of magnitude smaller effective attempt frequencies other than the universal one of $\nu_{0}=10^{13} \mathrm{~s}^{-1}$ for systems with $E_{m}<100 \mathrm{meV}$. This is associated with finite barrier effects at the employed temperatures, where $k T$ amounts to a substantial fraction of $E_{m}$, as will be detailed in a forthcoming publication.

[19] The slope of $\log \left[n_{x}(1 / T)\right]$ for $T>200 \mathrm{~K}$ is determined exclusively by $\Delta E$. The interaction range is not critical, provided $E_{\mathrm{d} l \uparrow}$ and $E_{\mathrm{d} \downarrow \downarrow}$ are modified such that $\Delta E$ rests constant.

[20] J. W. Evans and M. C. Bartelt, J. Vac. Sci. Technol. A 12, 1800 (1994). 